\title{
Fiducial Region Estimation of Parameter of Two Parameters Exponential Distribution
}

\author{
Xiuzhen $\mathrm{Li}^{1}$, Yanying $\mathrm{Ma}^{1, \mathrm{a}^{*}}$, Chunguang Huang ${ }^{2}$ and Xin $\mathrm{Wang}^{3}$ \\ ${ }^{1}$ Jilin Engineering Normal University .Changchun; China; \\ ${ }^{2} \mathrm{NO} .11$ High school of Changchun Jilin China \\ ${ }^{3}$ Shenyang Jianzhu University. Shenyang, China \\ a2238552865@qq.com \\ ${ }^{*}$ corresponding author
}

Keywords: Exponential distribution; Fiducial distribution; Region estimation; Combination fiducial region

\begin{abstract}
Exponential distribution is very important distribution, it is often used to approximate various life distributions, and is widely used in in the areas of queuing theory and reliability theory. The article studied the combination fiducial region estimation of two parameters exponential distribution under the study of fiducial interval estimation of single parameter of two parameters exponential distribution.
\end{abstract}

\section{Introduction}

\section{Definition of two parameters exponential distribution}

Definition: Suppose the random variable $\mathrm{x}$ obey the two parameters exponential distribution . It's distribution function and distribution density function is

$$
F(x ; \mu, \sigma)=1-e^{-\frac{x-\mu}{\sigma}} ; \quad f(x ; \mu, \sigma)=\frac{1}{\sigma} e^{-\frac{x-\mu}{\sigma}}
$$

Which $x \geq \mu, \sigma>0, \mu$ is positional parameter, $\sigma$ is scale parameter,then we called the random variable $\mathrm{x}$ obey the two parameters exponential distribution .often note: $x \sim E(\mu, \sigma)$

\section{Fundamental lemma}

Suppose $X_{1}, X_{2}, \ldots \ldots X_{n}$ are Simple random samples with capacity of $\mathrm{n}$ that from exponential distribution, then we have order statistic $X_{(1)} \leq X_{(2)} \leq \ldots \leq X_{(n)}$, obtained sample observations $x_{1}, x_{2}, \ldots x_{n}$,and then the maximum likelihood estimation of $\mu$ and $\sigma^{2}$ are obtained

$$
\begin{aligned}
& \hat{\mu}=X_{(1)}=\min \left\{X_{1}, X_{2}, \ldots X_{n}\right\} \\
& \hat{\sigma}=\bar{X}-X_{(1)}
\end{aligned}
$$

In order to study the problem, convenience,the following four lemmas are given

Lemma 1: Suppose $X_{(1)} \leq X_{(2)} \leq \ldots \leq X_{(r)}$ is the first $\mathrm{r}$ order statistics $(1 \leq r \leq n)$ that from population $E(\mu, \sigma)$, then

$$
\mathrm{Y}=\frac{2 n\left(X_{(1)}-\mu\right)}{\sigma} \sim \chi^{2}
$$

Lemma 2: Suppose $X_{(1)} \leq X_{(2)} \leq \ldots \leq X_{(r)}$ is the first $r$ order statistics that from population $E(\mu, \sigma)$ that from population $E(\mu, \sigma)$

Remember $Y_{1}=n X_{(1)}, Y_{2}=(n-1)\left(X_{(2)}-X_{(1)}\right), \ldots Y_{i}=(n-i+1)\left(X_{(i)}-X_{(i-1)}\right), i=2,3 \ldots, \mathrm{n}$, then We have the following conclusions

(1) $\sum_{i=1}^{n} X_{(i)}=\sum_{i=1}^{n} Y_{i}$ 
(2) $Y_{1}, Y_{2}, \ldots Y_{n}$ independent of each other

(3) $Y_{1} \sim E\left(n \mu_{n} \sigma\right), Y_{2}, Y_{3} \ldots Y_{n}$ are the same as single parameter exponential distribution $E(\sigma)$

Lemma 3 : Suppose $Z_{1}, Z_{2}, \ldots Z_{n}$ independent and the same distribution as $E(\sigma)$, then $Z_{1}+Z_{2}+\ldots+Z_{n} \sim G a\left(n, \frac{1}{\sigma}\right)$

Lemma 4: Suppose $\mathrm{Z} \sim G a\left(n, \frac{1}{\sigma}\right)$, then $\frac{2 Z}{\sigma} \sim \chi^{2}(2 \mathrm{n})$.

\section{Building function model,Induced fiducial distribution}

Extract a simple random sample $X_{1}, X_{2}, \ldots X_{n}$ from the population $E(\mu, \sigma)$,obtain the first $\mathrm{r}$ order statistics $X_{(1)} \leq X_{(2)} \leq \ldots \leq X_{(r)}(1 \leq r \leq n)$. By Lemma 1 we can know

$$
\frac{2 n\left(X_{(1)}-\mu\right)}{\sigma} \sim \chi^{2}(2)
$$

Note $Y_{i}=(n-i+1)\left(X_{(i)}-X_{(i-1)}\right) \quad i=2,3 \ldots$, n. then

$$
n\left(\bar{X}-X_{(1)}\right)=\sum_{i=1}^{n} X_{(i)}-n X_{(1)}=\sum_{i=1}^{n} Y_{i}-Y_{1}=\sum_{i=2}^{n} Y_{i}
$$

By Lemma 2 we can know: $Y_{2}, \ldots Y_{n}$ is equally distributed in Single parameter exponential distribution $E(\sigma)$,then by Lemma 3 we can obtain:

$$
n\left(\bar{X}-X_{(1)}\right)=\sum_{i=2}^{n} Y_{i}=Y_{2}+Y_{3}+\ldots Y_{n} \sim G a\left(n-1, \frac{1}{\sigma}\right)
$$

Also due to $\frac{2}{\sigma} \sum_{i=2}^{n} Y_{i}=\frac{2 n\left(\bar{X}-X_{(1)}\right)}{\sigma}$

By Lemma 4 we can obtain: $\frac{2 n\left(\bar{X}-X_{(1)}\right)}{\sigma} \sim \chi^{2}(2(\mathrm{n}-1))$

Note: $\left\{\begin{array}{c}e_{1}=\frac{2 n\left(X_{(1)}-\mu\right)}{\sigma} \sim \chi^{2}(2) \\ e_{2}=\frac{2 n\left(\bar{X}-X_{(1)}\right)}{\sigma} \sim \chi^{2}(2(n-1))\end{array}\right.$

Because $e_{1}$ is a function of $X_{(1)}, e_{2}$ is a function of $\left(\mathrm{X}-X_{(1)}\right)$ yet $X_{(1)}$ and $\left(\mathrm{X}-X_{(1)}\right)$ independent of each other,so $e_{1}$ and $e_{2}$ independent of each other too, so we can obtain function model:

$$
\left\{\begin{array}{l}
X_{(1)}=\frac{\sigma}{2 n} e_{1}+\mu \\
\bar{X}-X_{(1)}=\frac{\sigma}{2 n} e_{2}
\end{array}\right.
$$

In this function model, $\left(X_{(1)}, \mathrm{X}-X_{(1)}\right)$ is sample observations, $(\mu, \sigma)$ is parameter vector, $\left(e_{1}, e_{2}\right)$ is error variance,so by (9) we can obtained the following conclusion

$$
\left\{\begin{array}{c}
\mu=X_{(1)}-\frac{e_{1}}{e_{2}}\left(\bar{X}-X_{(1)}\right) \\
\sigma=\frac{2 n\left(\bar{X}-X_{(1)}\right)}{e_{2}}
\end{array}\right.
$$
is

Because $e_{1}, e_{2}$ is independent of each other,by (8) we can obtain joint density function of $\left(e_{1}, e_{2}\right)$ 


$$
\begin{aligned}
h\left(e_{1}, e_{2}\right) & =\frac{1}{2} \exp \left\{-\frac{1}{2} e_{1}\right\} \frac{1}{2^{n-1} \Gamma(n-1)} e_{2}^{n-2} \exp \left\{-\frac{1}{2} e_{2}\right\} \\
& =\frac{1}{2^{n} \Gamma(n-1)} e_{2}^{n-2} \exp \left\{-\frac{1}{2}\left(e_{1}+e_{2}\right)\right\}
\end{aligned}
$$

According to the transformation formula of random vector,Do transform from $\left(e_{1}, e_{2}\right)$ to $(\mu, \sigma)$,Find the absolute value of the Jacobi determinant corresponding to (8)

$$
|J|=\left\|\begin{array}{|ll}
\frac{\partial e_{1}}{\partial \mu} & \frac{\partial e_{1}}{\partial \sigma} \\
\frac{\partial e_{2}}{\partial \mu} & \frac{\partial e_{2}}{\partial \sigma}
\end{array}\left|=\| \begin{array}{cc}
\sigma & \frac{-2 n\left(x_{(1)}-\mu\right)}{\sigma^{2}} \\
0 & \frac{-2 n\left(\bar{x}-x_{(1)}\right)}{\sigma^{2}}
\end{array}\right|=\left|\frac{4 n^{2}\left(\bar{x}-x_{(1)}\right)}{\sigma^{3}}\right|\right.
$$

By bringing in operations we can obtain the joint fiducial density function of $(\mu, \sigma)$

$$
\begin{aligned}
g(\mu, \sigma) & =\exp \left\{-\frac{n(\bar{x}-\mu)}{\sigma}\right\} \frac{1}{2^{n-2} \Gamma(n-1)}\left[\frac{2 n\left(\bar{x}-x_{(1)}\right)}{\sigma}\right]^{n-2} \cdot \frac{n^{2}\left(\bar{x}-x_{(1)}\right)}{\sigma^{3}} \\
& =\exp \left\{-\frac{n(\bar{x}-\mu)}{\sigma}\right\} \frac{1}{\Gamma(n-1)} \cdot \frac{n^{n}\left(\bar{x}-x_{(1)}\right)^{n-1}}{\sigma^{n+1}} \quad \mu<x_{(1)}, \quad \sigma>0
\end{aligned}
$$

On the open interval $\left(-\infty, x_{(1)}\right)$ The last formula for $\mu$ integral, the marginal fiducial density function of $\sigma$ can be obtained:

$$
\begin{aligned}
f(\sigma) & =\int_{-\infty}^{x_{(1)}} g(\mu, \sigma) d \mu \\
& =\exp \left\{-\frac{n\left(\bar{x}-x_{(1)}\right)}{\sigma}\right\} \frac{\left[n\left(\bar{x}-x_{(1)}\right)\right]^{n-1}}{\Gamma(n-1)} \cdot \sigma^{-n} \quad \sigma>0
\end{aligned}
$$

The same methods, the marginal fiducial density function of $\mu$ can be obtained

$$
\begin{array}{rlr}
\tau(\mu) & =\int_{0}^{\infty} g(\mu, \sigma) d \sigma \\
& =(n-1) \frac{\left(\bar{x}-x_{(1)}\right)^{n-1}}{(\bar{x}-\mu)^{n}} \quad\left(\mu<x_{(1)}\right)
\end{array}
$$

\section{The estimation of fiducial region (interval) is given}

According to ( 12$)$,For a given level of $1-\alpha(0<\alpha<1)$, Existence of $\mathrm{G}$ makes $\iint_{G} g(\mu, \sigma) d \mu d \sigma=1-\alpha$

Then the $\mathrm{G}$ is a joint fiducial region estimation of the two parameters $(\mu, \sigma)$,that the fiducial level is $1-\alpha(0<\alpha<1)$, Obviously, there's more than one of these $\mathrm{G}$, The following is a very special case. Although $\mu$ and $\sigma$ are not necessarily independent,but $e_{1} \sim \chi^{2}$ (2), $e_{2} \sim \chi^{2}$ (2(n-1)) Moreover $e_{1}, e_{2}$ is independent of each other

$$
e_{1}=\frac{2 n\left(X_{(1)}-\mu\right)}{\sigma} \quad, \quad e_{2}=\frac{2 n\left(\bar{X}-X_{(1)}\right)}{\sigma}
$$

For a given fiducial level $1-\alpha$, take the appropriate $a_{1}, a_{2}, b_{1}, b_{2}$ then

$\mathrm{P}\left(a_{1}<e_{1}<a_{2}, b_{1}<e_{2}<b_{2}\right)=1-\alpha$ because $e_{1}, e_{2}$ independent of each other, then

$\mathrm{P}\left(a_{1}<e_{1}<a_{2}\right) \mathrm{P}\left(b_{1}<e_{2}<b_{2}\right)=1-\alpha$

Arbitrary selection $0<\beta_{1}<1,0<\beta_{2}<1$, make $\beta_{1} \beta_{2}=1-\alpha$

Make $\mathrm{P}\left(a_{1}<e_{1}<a_{2}\right)=\beta_{1}, \mathrm{P}\left(b_{1}<e_{2}<b_{2}\right)=\beta_{2}$ 
Because $e_{1}$ and $e_{2}$ obeys $\chi^{2}$ distribution, The distribution density images are unimodal and asymmetric,in order to study the problem, convenience,Take the tail case (Note: It's also another the reason why the region of fiducial that is chosen here is not unique ). Take G's rectangle about $\left(e_{1}, e_{2}\right)$

Take $a_{1}=\frac{\chi_{\frac{1-\beta_{1}}{2}}^{2}}{2}(2) \quad, a_{2}=\frac{\chi_{\frac{1+\beta_{1}}{2}}^{2}}{2}(2) ; \quad b_{1}=\frac{\chi_{\frac{1-\beta_{2}}{2}}^{2}}{2}(2(n-1)), b_{2}=\frac{\chi_{\frac{1+\beta_{2}}{2}}^{2}}{2}(2(n-1))$

because

$$
\begin{aligned}
& \mathrm{P}\left(a_{1}<e_{1}<a_{2}, b_{1}<e_{2}<b_{2}\right) \\
& =\mathrm{P}\left\{\frac{a_{2} \sigma}{2 n}-X_{(1)}<\mu<\frac{a_{1} \sigma}{2 n}-X_{(1)}, \quad \frac{2 n\left(\bar{X}-X_{(1)}\right)}{b_{2}}<\sigma<\frac{2 n\left(\bar{X}-X_{(1)}\right)}{b_{1}}\right\}
\end{aligned}
$$

so,a fiducial region $\mathrm{G}$ of $(\mu, \sigma)$ that fiducial level is $1-\alpha \quad(0<\alpha<1)$ $\mathrm{G}=\left\{\frac{a_{2} \sigma}{2 n}-X_{(1)}<\mu<\frac{a_{1} \sigma}{2 n}-X_{(1)}, \quad \frac{2 n\left(\bar{X}-X_{(1)}\right)}{b_{2}}<\sigma<\frac{2 n\left(\bar{X}-X_{(1)}\right)}{b_{1}}\right\}$

Where $a_{1}, a_{2}, b_{1}, b_{2}$ is optional constant, $\beta_{1} \beta_{2}=1-\alpha$.

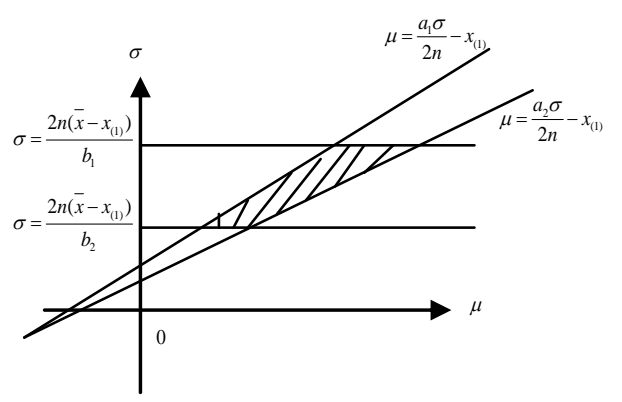

Figure 1. Fiducial region

\section{Acknowledgements}

This research was supported by the research and practice of Fiducial region estimation of parameter of two parameters exponential distribution (No.XYB201719), Jilin Science Planning Project on"The research and practice of integrating mathematical modeling into the basic course of Mathematics under the background of application type" (No.YB14-36) and the Support Foundation of Shenyang Jianzhu University (No.2017034) respectively.

\section{References}

[1] Mao Shi-song,Wang Jing-long,Pu Xiao-long. Advanced Mathematical Statistics [M]. higher education publishing house, 1998

[2] zhang Hong-bing, Liu Rui-yuan; Li jie. The shortest confidence interval of parameters of two parameter exponential distribution [J]. Journal of Neijiang Normal University,2007,12.

[3] Gao Shang.Calculation of shortest confidence interval[J].Journal of east china shipbuilding institute, 2003.

[4] Dawid A P and Stone M.The functional-model basis of fiducial inference（with discussion ）.Ann Statist, 1982,10:01054-1074

[5] Dawid AP and Wang J L. Fiducial prediction and semi-bayesion infetence. Ann Statist, 1993,21:1119-1138.

[6] Chen Xi-ru.Probability Theory and Mathematical Statistics [M].University of Science and Technology of China publishing house, 1992. 